\title{
Synchronization of discrete Sprott chaotic systems and its application in secure communication
}

\author{
Hang-Hong Kuo ${ }^{1}$, Teh-Lu Liao ${ }^{2}$, Jun-Juh $\mathrm{Yan}^{3 *}$, Yu-Chen $\mathrm{Hsu}^{3}$ \\ ${ }^{1}$ NeoVictory Technology Co., Ltd., Tainan 710, Taiwan \\ ${ }^{2}$ Department of Engineering Science, National Cheng Kung University \\ Tainan 701, Taiwan, R.O.C. \\ 3* Department of Computer and Communication, Shu-Te University, \\ Kaohsiung 824, Taiwan, (Tel: +886-7-6158000 \#4806) \\ *Corresponding Author: jjyan@stu.edu.tw
}

\begin{abstract}
This paper is concerned with the synchronization of discrete master-slave Sprott chaotic systems and its application in secure communication. First, we embed the message into the master discrete chaotic system. Then a discrete sliding mode scheme is utilized to solve the synchronization problem of discrete master-slave Sprott chaotic systems. The proposed scheme can guarantee the synchronization of systems based on the use of Lyapunov stability theory. The selection of discrete switching surface and the existence of discrete sliding mode are addressed. Finally, an illustrative example is given to demonstrate the validity of the proposed theoretical results.
\end{abstract}

Keywords: Synchronization, discrete Sprott chaotic systems, discrete sliding mode.

\section{Introduction}

Chaos phenomenon has been generally developed and studied over the past two decades. Chaotic systems exist particular properties, such as broadband noise-like waveform, and depending sensitively on the system's precise initial conditions, etc. [Gui et al. (2006)], [Chadli et al. (2014)], [Zhou et al. (2015)]. These properties offer some advantages for designing secure communication systems. Due to its powerful applications in engineering systems, both control and synchronization problems have extensively been studied in the past decades for analogue chaotic/hyperchaotic systems such as Lorenz system, Chua's system, Rössler system, Chen's system, Lur'e system, Sprott system and chaotic neural networks. Until now, many control methods for analogue chaotic systems have extensively been studied extensively in the literature, such as linear state observer design, impulsive control, adaptive control, sampled driving signal via Takagi-Sugeno (T-S) fuzzy model, continuous sliding mode control design, etc. [Agiza et al (2004)], [Elabbasy et al (2004)], [Rafikov et al (2004)], [Liao et al (2006)], [Liao et al (1999)], [Sun et al (2004)].

Sliding-mode control (SMC) is a characteristic kind of variable structure systems. In these two decades, sliding-mode control has been a useful and distinctive robust control strategy for many kinds of engineer systems. Depending on the proposed switching surface and discontinuous controller, the trajectories of dynamic systems can be guide to the fixed sliding manifold. The proposed performance on request can be satisfied. In general, there are two main advantages of SMC which are the reducing order of dynamics from the purposed switching functions and robustness of restraining system uncertainties. Many studies have been conducted on SMC [Chang et al. (2005)], [Jang et al. (2005)], [Utkin (1978)].

On the other hand, due to the progress of computer and DSP technology, using them to implement the controller has become more and more popular and important. Therefore, research in discrete-time control has become intensified in recent years, and it is quite natural to extend the method of continuous control to discrete-time systems. Over the past few years, it has been reported to many practical control systems. Several design methods of discrete-time SMC have been found in the literature [Hoz et al. (2014)], [Yang et al (1996)], [Chee et al. (2003)], [Wu et al. (2010)], [Zhou et al (2008)], [Yan et al. (2008)], [Lin et al. (2010)].

In this paper, we aim to design a secure communication system based on the synchronization of discrete master-slave Sprott chaotic systems. First, we embed the message into the master discrete chaotic system. Then the discrete sliding mode scheme is utilized to guarantee the synchronization of discrete master-slave Sprott chaotic systems. After achieving the synchronization, the embedded message in transmitter can be recovered in the receiver. Finally, an illustrative example is given to demonstrate the validity of the proposed theoretical results.

\section{System description and proposed theorem}


Consider the following Sprott circuit, which is one of typical chaotic systems.

$$
\dot{x}(t)=\left[\begin{array}{c}
\dot{x}_{1}(t) \\
\dot{x}_{2}(t) \\
\dot{x}_{3}(t)
\end{array}\right]=\underbrace{\left[\begin{array}{ccc}
0 & 1 & 0 \\
0 & 0 & 1 \\
-1.2 & -1 & -0.6
\end{array}\right]}_{A} x(t)+\underbrace{\left[\begin{array}{l}
0 \\
0 \\
1
\end{array}\right]}_{B} \underbrace{\left(2 k_{a} \operatorname{sign}\left(x_{1}(t)\right)\right.}_{g(x(t))}+m(t))
$$

where $\dot{x}$ denote the derivative of $x$. The constant parameter $k_{a}>0$ is used to adjust the amplitude of the response chaotic system and this parameter does not affect the chaotic behaviour of the original system. The $m(t)$ is the embedded message bounded by $|m(t)| \leq r, \quad r>0$.

Remark 1: Consider continuous chaotic dynamic systems described by

$$
\dot{x}(t)=A x(t)+B g(x(t), m(t))
$$

where $x(t)$ is the state vector, $g(x(t), m(t)) \in R^{m}$ represents the nonlinear vector, The matrices $A$ and $B$ are known constant matrices of appropriate dimensions. The discrete-time representation of system (2) with sample and hold process is given by

$$
x_{d}(k+1) T=G x_{d}(k T)+H g\left(x_{d}(k T)\right)
$$

where $T$ is the sampling time, $G=e^{A T} ; H=\left[G-I_{n}\right] A^{-1} B$, and assume $(G, H)$ is controllable.

Based on the master-slave concept for synchronization of the systems, the discrete master and slave Sprott circuits are described by the following differential equations (4) and (5), respectively. When sampling time $T=0.001 \mathrm{sec}$, then the master and slave discrete dynamic systems via (3) can be obtained, respectively.

$$
\begin{aligned}
& x_{m d}(k+1) T=\left[\begin{array}{c}
x_{m d 1}(k+1) T \\
x_{m d 2}(k+1) T \\
x_{m d 3}(k+1) T
\end{array}\right] \\
& =\left[\begin{array}{ccc}
1 & 0.001 & 0 \\
0 & 1 & 0.001 \\
-0.0012 & -0.001 & 0.9994
\end{array}\right] x_{m d}(k T) \\
& +\left[\begin{array}{c}
0 \\
0 \\
0.001
\end{array}\right]\left(2 k_{a} \operatorname{sign}\left(x_{m 1}(k T)\right)+m(k T)\right)
\end{aligned}
$$

and

$$
\begin{aligned}
& x_{s d}(k+1) T=\left[\begin{array}{c}
x_{s d 1}(k+1) T \\
x_{s d 2}(k+1) T \\
x_{s d 3}(k+1) T
\end{array}\right]= \\
& {\left[\begin{array}{ccc}
1 & 0.001 & 0 \\
0 & 1 & 0.001 \\
-0.0012 & -0.001 & 0.9994
\end{array}\right] x_{s d}(k T)} \\
& +\left[\begin{array}{c}
0 \\
0 \\
0.001
\end{array}\right]\left(2 k_{a} \operatorname{sign}\left(x_{m 1}(k T)\right)+u(k T)\right)
\end{aligned}
$$

The dynamics of synchronization error $e(k+1) T=x_{m d}(k+1)-x_{s d}(k+1)$ between the master-slave systems given in (4) and (5) can be described by the following compact form:

$$
\begin{array}{r}
e(k+1) T=\left[\begin{array}{c}
e_{1}(k+1) T \\
e_{2}(k+1) T \\
e_{3}(k+1) T
\end{array}\right]=\left[\begin{array}{ccc}
1 & 0.001 & 0 \\
0 & 1 & 0.001 \\
-0.0012 & -0.001 & 0.9994
\end{array}\right] e(k T) \\
+\left[\begin{array}{c}
0 \\
0 \\
0.001
\end{array}\right](m(k T)-u(k T))
\end{array}
$$

It is clear that the synchronization problem between (4) and (5) becomes the equivalent problem of stabilization the error dynamics (6). In the presence of control function, the synchronization between two discrete Sprott models (4) and (5) may occur provided the error state can converge to zero, i.e.

$$
\lim _{k \rightarrow \infty}\|e(k T)\|=\lim _{k \rightarrow \infty}\left\|x_{m d}(k T)-x_{s d}(k T)\right\| \rightarrow 0, \quad i=1,2,3 .
$$

Here discrete SMC design for secure communication system is to guarantee that the system can reach onto and maintain subsequently on the sliding surface. The designed switching surface $s(k)$ is given as follows:

$$
s(k)=a_{1} e_{1}(k)+a_{2} e_{2}(k)+e_{3}(k)
$$

where $s \in R$ and $a_{i} \in R$ for $i=1,2$ are designed constants. When the system operates in the sliding mode, the following equation must be satisfied

$$
\begin{aligned}
& s(k)=a_{1} e_{1}(k)+a_{2} e_{2}(k)+e_{3}(k)=0 \\
& e_{3}(k)=-a_{1} e_{1}(k)-a_{2} e_{2}(k)
\end{aligned}
$$

Thus error dynamics become

$$
\begin{aligned}
& {\left[\begin{array}{l}
e_{1}(k+1) \\
e_{2}(k+1)
\end{array}\right]=\left[\begin{array}{cc}
1 & 0.001 \\
-0.001 * a_{1} & 1-0.001 * a_{2}
\end{array}\right]\left[\begin{array}{l}
e_{1}(k) \\
e_{2}(k)
\end{array}\right]} \\
& =A\left[\begin{array}{l}
e_{1}(k) \\
e_{2}(k)
\end{array}\right]
\end{aligned}
$$

Obviously, if the constants $a_{i}$ are selected such that the eigenvalues of matrix $A$ in (11) is located in unit circle, then the above error dynamics is exponentially stable Also the convergence rate can be determined by the eigenvalues of matrix $A$. Furthermore, $e_{3}$ converges to zero when $e_{1}$ and $e_{2}$ converge to zero. After establishing an appropriate sliding surface, the next step is to design a discrete sliding mode controller to drive the system trajectories onto the sliding surface $s(k)=0$.

Lemma 1: [Yan et al (2008)] For a discrete sliding mode control system, if it satisfies the following hitting conditions of sliding motion, then the trajectories of the controlled dynamics system converge to the sliding mode $s(k)=0$. 


$$
\begin{cases}\Delta s_{k}=s(k+1)-s(k) \leq-\varepsilon T \operatorname{sign}(s(k))-q T s(k), & \text { if } s(k)>0 \\ \Delta s_{k}=s(k+1)-s(k) \geq-\varepsilon T \operatorname{sign}(s(k))-q T s(k), & \text { if } s(k)<0\end{cases}
$$

where $q>0 ; \varepsilon>0 ; 1-q T>0 ; T>0$ the sampling period $。$

Theorem 1: If the control input $u(t)$ is designed as:

$$
u(k)=-u_{1}-(\varepsilon+r) \cdot \operatorname{sign}(s(k))-q s(k) ;
$$

where $u_{1}=-1.2 e_{1}(k)-\left(a_{1}-1\right) e_{2}(k)-\left(a_{2}+0.6\right) e_{3}(k)$, then reaching condition of the sliding mode is satisfied and the trajectories of the controlled dynamics system converge to the sliding mode $s(k)=0$.

\section{Proof:}

$$
\begin{aligned}
\Delta s_{k} & =s(k+1)-s(k) \\
& =e_{3}(k+1)+a_{2} e_{2}(k+1)+a_{1} e_{1}(k+1)-e_{3}(k)-a_{2} e_{2}(k)-a_{1} e_{1}(k) \\
& =T u_{1}(k)+\operatorname{Tm}(k)+T u(k) \\
& =\operatorname{Tm}(k)-(\varepsilon+r) T \cdot \operatorname{sign}(s(k))-q T s(k)
\end{aligned}
$$

Obviously

$$
\begin{cases}\operatorname{Tm}(k)-r T \cdot \operatorname{sign}(s(k)) \leq 0, & \text { if } s(k)>0 \\ \operatorname{Tm}(k)-r T \cdot \operatorname{sign}(s(k)) \geq 0, & \text { if } s(k)<0\end{cases}
$$

Therefore, the hitting condition is always satisfied and $s(k)$ always converges to the switching surface $s=0$. Hence the proof is achieved completely.

Having ensured the error dynamics converges to zero as discussed above, we can further obtain following result:

$$
\begin{aligned}
& e_{1}(k+1)=0 \\
& e_{2}(k+1)=0 \\
& e_{3}(k+1)=0+0.001(u(k)-m(k))=0
\end{aligned}
$$

Then, we can deduce that $\lim _{k \rightarrow \infty}(u(k)-m(k))=0$

It means that the message $m(k)$ can be recovered in the receiver from the control input $u(k)$. In order to avoid the chattering occurs, we can use the equivalent control with saturation function to replace the sign function [Lin et al. (2010)].

$$
u_{e q}(k)=-u_{1}-(\varepsilon+r) \cdot \frac{s(k)}{|s(k)|+\delta}-q s(k) ;
$$

where $\delta$ is an arbitrarily small positive constant. When we choose $\delta$ enough small, then the control input $u(k)$ can be approximated by the following equivalent control $u_{e q}(k)$.

\section{Experiment results}

In this section, both simulation and experimental results are presented to demonstrate the effectiveness of the proposed secure communication system. For simulation, we embed a $\sin$ wave $m(t)=\sin (\pi t)$ bounded by $|m(t)| \leq 1$ into the dynamics of master system and the parameter $k_{a}=3$. And then, as described above, the proposed design procedure can be summarized as follows:

Step1: According to (11), $a_{1}=701000, a_{2}=900$ are chosen such that the eigenvalues of matrix $A$ are $(0.5,-0.4)$ to result in a stable sliding motion. Therefore, the switching surface is obtained as

$$
s(k)=701000 e_{1}+900 e_{2}+e_{3}
$$

Step2: According to (17), the sliding mode control law is obtained as follows:

$$
\begin{aligned}
& u_{e q}(k)=-u_{1}-(\varepsilon+r) \cdot \frac{s(k)}{|s(k)|+\delta}-q s(k) ; \\
& \varepsilon=1, \delta=0.01, q=3, r=1
\end{aligned}
$$

where $u_{1}=-1.2 e_{1}(k)-700999 e_{2}(k)-900.6 e_{3}(k)$.

The simulation results with initial conditions $\left[\begin{array}{lll}x_{m 1}(0) & x_{m 2}(0) & x_{m 3}(0)\end{array}\right]=\left[\begin{array}{lll}1 & -0.05 & -0.5\end{array}\right] \quad$ and $\left[x_{s 1}(0) \quad x_{s 2}(0) \quad x_{s 3}(0)\right]=\left[\begin{array}{lll}1 & -0.02 & -1\end{array}\right]$ are shown in Figs. 1-2. The chaotic attractor of discrete Sprott chaotic system is given in Fig. 1. The system state responses trajectory of controller design is shown in Fig. 2. When $t=k T=0.09 \mathrm{sec}$., it is obvious that the controller guarantee synchronization of discrete Sprott chaotic systems. From the simulation results, it is shown that the proposed controller works well to guarantee synchronization. Figure 3 displays that the sliding mode surface $s(t)$ converges to zero under the proposed control.

\section{Conclusions}

In this paper, we have designed a secure communication system based on the synchronization of discrete master-slave Sprott chaotic systems. By applying discrete sliding mode control and Lyapunov stability theory, the problem of synchronization for discrete Sprott chaotic systems its application in secure communication have been investigated. An illustrative example has demonstrated the validity of the proposed theoretical results.

\section{References}

Gui, A. and Ge, W. (2006). Periodic solution and chaotic strange attractor for shunting inhibitory cellular neural networks with impulses, CHAOS, 16, 033116.

Chadli, M. Zelinka, I. (2014). Chaos synchronization of unknown inputs Takagi-Sugeno fuzzy: Application to secure communications, Computers and Mathematics with Applications, $68,2142-2147$

Zhou, Lili, Wang, C., He, H., Lin, Y. (2015). Time-controllable combinatorial inner synchronization and outer synchronization of anti-star networks and its application in secure 
communication," Commun. Nonlinear Sci. Numer. Simulat, 22: 623-640.

Park, J. H. (2005). Adaptive synchronization of a unified chaotic system with an uncertain parameter, International Journal of Nonlinear Sciences and Numerical Simulation, 6, 201-206.

Lian, K. Y., Chiang, T. S., Chiu, C. S., and Liu, P. (2001a). Synthesis of fuzzy mode-based designs to synchronization and secure communications for chaotic systems, IEEE Transactions on Systems, Man, and Cybernetics Part B, 31, 66-83.

Lian, K. Y., Chiu,C. S., Chiang, T. S., and Liu, P. (2001b). Secure communications of chaotic systems with robust performance via fuzzy observer-based design," IEEE Transactions on Fuzzy Systems, 9, 212-220.

Agiza, H. N. (2004). Chaos synchronization of Lü dynamical system, Nonlinear Analysis, 58, 11-20.

Elabbasy, E. M., Agiza, H. N., and El-Dessoky, M. M. (2004). Controlling and synchronization of Rossler system with uncertain parameters, International Journal of Nonlinear Sciences and Numerical Simulation, 5, 171-181, 2004.

Rafikov, M., and Balthazar, J. M. (2004). On an optimal control design for Rössler system, Physics Letter A, 333, 241-245.

Liao, X. and Yu, P. (2006). Chaos control for the family of Rössler systems using feedback controllers, Chaos, Solitons \& Fractals, 29, 91-107.

Liao, T. L. and Huang, N. S. (1999). An observer-based approach for chaotic synchronization with applications to secure communications, IEEE Transactions on Circuits Systems I, 46, $1144-1150$.

Sun, J., and Zhang, Y. (2004). Impulsive control and synchronization of Chua's oscillators, Mathematics Computers in Simulation, 66, 499-508.

Chang, W. D., and Yan, J. J. (2005). Adaptive robust PID controller design based on a sliding mode for uncertain chaotic systems, Chaos, Solitons \& Fractals, 26, 167-175.

Jang, M. J., Chen, C. C., and Chen, C. O. (2002). Sliding mode control of chaos in the cubic Chua's circuit system, International Journal of Bifurcation and Chaos, 12, 1437-1449.

Utkin, V. I. (1978). Sliding Mode and Their Applications in Variable Structure Systems, Moscow: Mir Editors.

Hoz, M.Z.D., and Acho, L., Vidal, Y. (2014). A modified Chua chaotic oscillator and its application to secure communications, Applied Mathematics and Computation, 247, $712-722$

Yang, T., and Chua, L. O. (1996) Secure communication via chaotic parameter modulation, IEEE Transactions on Circuits and Systems I, 43, 817-819.

Chee, C. Y., and Xu, D. (2003). Control of the formation of projective synchronization in lower-dimensional discrete-time systems, Phys Letter A, 318, 112-118.

Wu. J., Shien, L. S., Zhanh, Y., and Song, G. (2010). Digital controller design for Bouc-Wen model with high-order hysteretic nonlinearities through approximated scalar sign function, International Journal of Systems Science, 2010,10, 1581-1519.

Zhou, H. Q., Shieh, L. S., Liu, C. R., Wang, Q. G. (2008). Digital design of combined PI and state feedback controller for non-linear stochastic systems, IMA Journal of Mathematical Control and Information, 25, 305-322.

Yan, M. and Shi, Y. (2008). Robust discrete-time sliding mode control for uncertain systems with timevarying state delay, IET control theory and applications, 2, 662-674.

Lin, J. S., Huang, C. F. Liao, T. L. and Yan, J. J. (2010). Design and implementation of digital secure communication based on synchronized chaotic systems, Digital Signal Processing, 20, 229-237
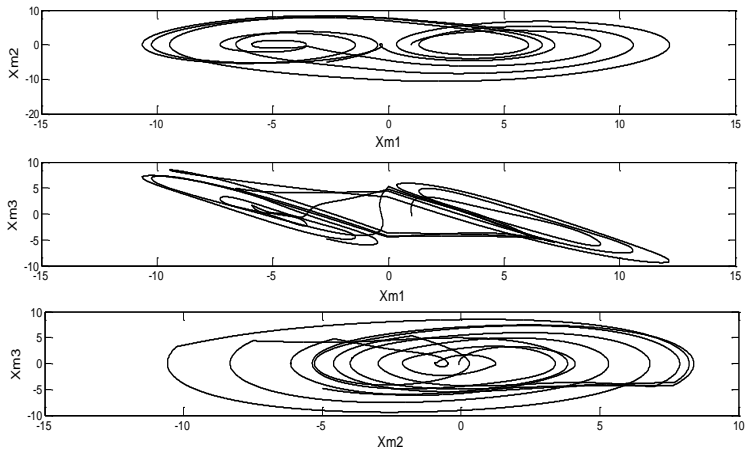

Figure 1. Chaotic behavior of discrete Sprott chaotic system.
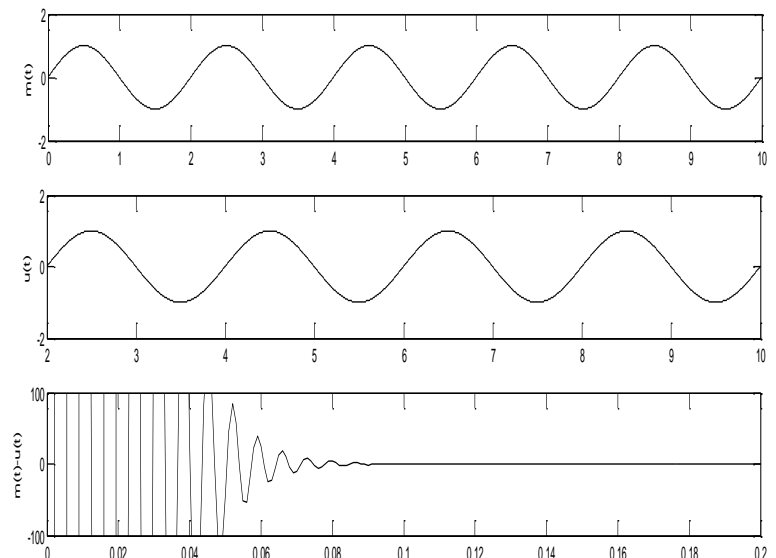

Figure 2. Time responses of message $m(t)$, controller $u(t)$, and synchronization $\mathrm{m}(\mathrm{t})-\mathrm{u}(\mathrm{t})$.

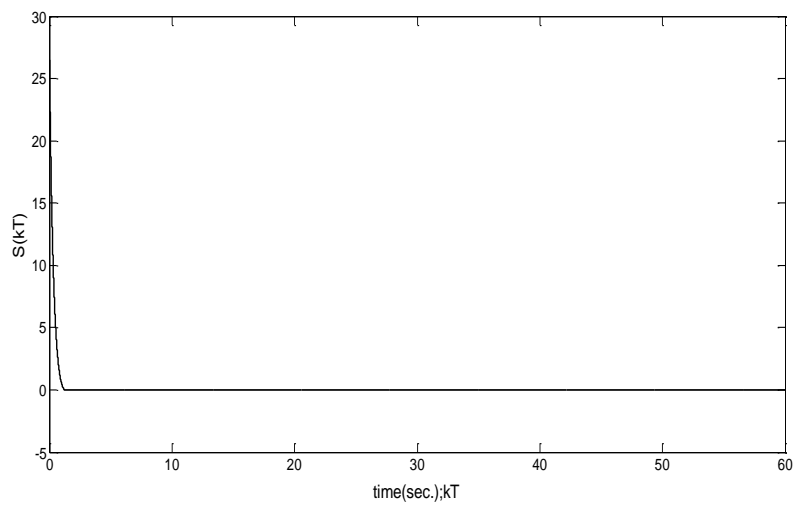

Figure 3. Time response of sliding mode surface $s(k T)$ 